\title{
Proposed Intelligent Pre-Processing Model of Real-Time Flood Forecasting and Warning for Data Classification and Aggregation
}

\author{
https://doi.org/10.3991/ijoe.v13i11.7382 \\ Marouane El Mabrouk $\left.{ }^{\varpi}\right)$, Salma Gaou \\ Abdelmalek Essaadi University, Tangier, Morocco \\ elmabroukmarouane@gmail.com
}

\begin{abstract}
A wireless sensor network is a network that can design a selforganizing structure and provides effective support for several protocols such as routing, locating, discovering services, etc. It is composed of several nodes called sensors grouped together into a network to communicate with each other and with the base stations. Nowadays, the use of Wireless sensor networks increased considerably. It can collect physical data and transform it into a digital values in real-time to monitor in a continuous manner different disaster like flood. However, due to various factors that can affect the wireless sensor networks namely, environmental, manufacturing errors hardware and software problems etc... It is necessary to carefully select and filter the data from the wireless sensors since we are providing a decision support system for flood forecasting and warning. In this paper, we presents an intelligent Pre-Processing model of real-time flood forecasting and warning for data classification and aggregation. The proposed model consists on several stages to monitor the wireless sensors and its proper functioning, to provide the most appropriate data received from the wireless sensor networks in order to guarantee the best accuracy in terms of real-time data and to generate a historical data to be used in the further flood forecasting.
\end{abstract}

Keywords-wireless sensor networks, multi-agent systems, data, classification, aggregation

\section{Introduction}

Flood disaster is the rise of watercourse waters, it is due to precipitation in large quantities, to which can be added a soil impermeable or became impermeable due to a drought important: the soil no longer absorbs the quantity of water that reaches it [1]. However, this may also be accentuated by direct and indirect causes like drainage, soil waterproofing, climate change etc. [2]. Nowadays, facing floods become a challenge for humans, that's why humans tried to anticipate and to forecast floods before its incidence in order to take all necessary precautions.

With technological development, wireless communication networks has been increasingly developed. Since their inception, it have been increasingly successful in the 
scientific and industrial communities. Thanks to its various advantages, this technology has been able to establish itself as an essential actor in the current network architectures [3]. In order to overcome floods, researchers have tried to obtain and acquire physical data in an automatic way in real-time. Thanks to this need, now we can benefit of the wireless sensors networks.

A wireless sensor network is a network composed of a set of embedded processing units, called "sensors", communicating via wireless links [4]. These wireless sensors are equipped with standard transmitters measuring instruments that convert the signals from radio transmission in process control instruments [5]. Despite its many advantages, wireless sensors networks has still major problems in terms of data acquisition and processing [6].

The classification approach was among the most suitable solutions to overcome this problem. Data classification is an approach that consists on organizing data into categories and into same specification [7]. Thanks to this process, data become easier to retrieve and to access. This approach requires tagging data in order to make easy the searching, eliminating duplication and getting the most accurate data [8].

The wireless sensor networks, the wireless communication and the data classification approach has significantly improved with the appearance and the utilization of the multi-agent systems [9]. A multi-agent system is an environment that has multiple agents communicating and collaborating between them to achieve the final result in a distributed way [10]. Thanks to the distribution property, that has the multi-agents systems, the WSN could benefit of this property to do complex processing and to do the classification process.

In this paper, we will present a new proposed intelligent pre-processing model of real-time flood forecasting and warning data received from the wireless sensors in order to do the classification and the aggregation of data using multi-agents system.

The rest of this paper is organized as follow:

The section 2 introduces the proposed model for classification and aggregation in wireless sensor networks by other researchers in the literature. The section 3 presents our new proposed intelligent pre-processing model of real-time flood forecasting and warning for data classification and aggregation, which consists on several stages to do this process. The section 4 introduces the design and the implementation of our proposed model. The results are presented in section 5, finally the Conclusion, and the future work in Section 6.

\section{Related Work}

There are many researches in the literature presented by other researchers in the field of the data classification in wireless sensor networks.

In [11], the authors of this paper proposed an energy-efficient data acquisition and classification scheme using Bloom filter. The authors proposed an extended Bloom filter method to fit their proposition. The authors used also a reservation-based Time Division Multiple Access protocol in order to reduce energy consumption. 
In [12], the authors proposed a new hierarchical clustering algorithm based on spectral classification in order to get accurate data and extend the lifetime and the energy of the wireless sensors. This algorithm has to stages; the first stage is for dividing and partitioning the wireless sensors into clusters, which contains some of sensors of the WSN. The second stage is for selecting a cluster head according to the energy of the sensor and its distance from the base station in order to communicate with the latter. The authors mentioned that using their algorithm reduce effectively the energy consumption of the sensor nodes and improve their lifetime according to the simulation and the results that they made.

In [13], the authors proposed a new adaptive preprocessing model for managing the received streaming data using a preprocessor and a predictor in order to increase the forecasting accuracy. The system two units, PCA (Principal Component Analysis) as preprocessor and Hyperbolic Hopfield Neural Network (HHNN) as predictor. According to the authors, by using this model, it provides an efficient and adaptive preprocessing of streaming data.

In [14], the authors proposed an adaptive technique for classification process to reduce the energy consumption of the sensor nodes and improve their lifetime. According to the authors, the strength of their proposed technique is that the functions are aware of the environmental situation changes. The authors proposed also an adaptive model for the acquisition and the utilization of the sensor data.

In [15], the authors proposed model that combines three different methods of machine learning, which are Parzen Windows, Identifying Densitybased Local Outliers and Outlier Detection With Active Learning for classifying data received from the wireless sensors to overcome the outlier fault in the wireless sensor networks. The authors made the validation and the simulation using real data acquired from motes deployed in an actual living lab.

In [16], the authors proposed a classifier for the Human activity recognition systems (HAR) using hybrid classification. In their paper, they presented the classification techniques (Un-supervised and the supervised) used to classify data received from the sensors placed in the human body to identify the activities made by these human. The authors made a comparison between the different presented classifiers presented in their paper with their proposed classifier in terms of accuracy. According to the authors, the results made showed that their proposed classifier is more accurate and efficient compared to the classification approaches in the literature.

\section{Proposed Intelligent Pre-Processing Model of Real-Time Flood Forecasting and Warning for Data Classification and Aggregation [17]}

The pre-processing phase is used to monitor the function and performance of sensors installed in flood-prone areas. This model is based on multi-agent systems and is used to classify the data received into valid and invalid data. We have proposed three stages with five agents to perform the necessary processing to classify the data to be 
stored in the database. In the next section, we will describe and detail the different stages of this proposed model.

\subsection{Sensor Verification Stage}

This stage consists of identifying which sensors are not working before beginning the classification phase. This process ensures the proper functioning of the sensors; if any sensor is broken, the system sends warning messages, text messages (SMS) and emails to managers advising them to repair or replace the sensors, which requires contacting the maintenance service. The verification agent is responsible for the process of verification and for monitoring the sensors' functioning. They are also responsible for sending ACL messages to the agents of the second stage, the classification stage, to trigger it.

\subsection{Data Aggregation and Classification Stage}

In this stage, three agents are responsible for the classification process, the Rainfall Agent, the Runoff Agent and the Water Level Agent. Each agent calculates a square matrix using a proposed formula to perform the comparison and classification as follows:

$$
M(C)=\left(\begin{array}{ccccccc}
0 & & & & & \\
T_{\left(d_{2}, d_{1}\right)} & 0 & & & & \\
T_{\left(d_{3}, d_{1}\right)} & T_{\left(d_{3}, d_{2}\right)} & 0 & & & \\
T_{\left(d_{4}, d_{1}\right)} & T_{\left(d_{4}, d_{2}\right)} & T_{\left(d_{4}, d_{3}\right)} & 0 & & \\
\mathrm{M} & \mathrm{M} & \mathrm{M} & 0 & 0 & \\
T_{\left(d_{n}, d_{1}\right)} & T_{\left(d_{n}, d_{2}\right)} & T_{\left(d_{n}, d_{3}\right)} & \mathrm{L} & T_{\left(d_{n}, d_{n-1}\right)} & 0
\end{array}\right)
$$

Where

$$
T_{\left(d_{i}, d_{j}\right)}=\left(1-\frac{\min \left(d_{i}, d_{j}\right)}{\max \left(d_{i}, d_{j}\right)}\right) \text { Where }\left\{\begin{array}{c}
i \in[2, n] \text { Where } n \in \bullet \\
j \in[1, n-1] \text { Where } n \in \bullet
\end{array}\right.
$$

The $d_{i}$ and $d_{j}$ are ones data from the dataset sent by sensors to be processed in the proposed matrix in position $i$ and $j . n$ is the number of lines and columns in the matrix.

We also proposed a parameter called the tolerance percentage. This percentage sets a tolerance margin for the data classification, and it allows us to identify the data that are in the range of tolerance and the data not in it. Finally, the valid and erroneous data are obtained, and the required classification is performed. The proposed formula for calculating this percentage is shown below: 


$$
P_{T C}=\frac{\left(2 \times \Delta_{C}\right)+T_{F}}{100}
$$

Where

- $\Delta_{C}$ : Sensor uncertainty designated by the fabrication company.

- $T_{F}$ : Uncertainty that may be attached to a factor in the study area (Rainfall, Runoff, ...)

\subsubsection{Application}

$E=\left\{V_{\text {Rainfall }}, V_{\text {Runoff }}, V_{\text {WaterLevel }}\right\}$ is the set of vectors for all data received from the sensors installed in the area. $V_{\text {Rainfall }}=\{80,82,81,84,90\}$ is a vector of five pieces of rainfall data sent by the rainfall sensor, $V_{\text {Runoff }}=\{30,78,73,77,70\}$ is a vector of five pieces of runoff data sent by the runoff sensor and $V_{\text {WaterLevel }}=\{40,45,20,41,42\}$ is a vector of five pieces of water level data sent by the water level sensor at the instant $t$.

We have $V_{\text {Rainfall }}=\{80,82,81,84,90\}$, so $n=5$.

Thus the Rainfall Matrix is,

\begin{tabular}{|c|c|c|c|c|c|}
\hline \multicolumn{7}{|c|}{ Rainfall Matrix M(C) } \\
\hline & $\mathbf{8 0}$ & $\mathbf{8 2}$ & $\mathbf{8 1}$ & $\mathbf{8 4}$ & $\mathbf{9 0}$ \\
\hline $\mathbf{8 0}$ & $\mathbf{0}$ & $\#$ & $\#$ & $\#$ & $\#$ \\
\hline $\mathbf{8 2}$ & $1-\frac{\min (82,80)}{\max (82,80)}$ & $\mathbf{0}$ & $\#$ & $\#$ & $\#$ \\
\hline $\mathbf{8 1}$ & $1-\frac{\min (81,80)}{\max (81,80)}$ & $1-\frac{\min (81,82)}{\max (81,82)}$ & $\mathbf{0}$ & $\#$ & $\#$ \\
\hline $\mathbf{8 4}$ & $1-\frac{\min (84,80)}{\max (84,80)}$ & $1-\frac{\min (84,82)}{\max (84,82)}$ & $1-\frac{\min (84,81)}{\max (84,81)}$ & $\mathbf{0}$ & $\#$ \\
\hline $\mathbf{9 0}$ & $1-\frac{\min (90,80)}{\max (90,80)}$ & $1-\frac{\min (90,82)}{\max (90,82)}$ & $1-\frac{\min (90,81)}{\max (90,81)}$ & $1-\frac{\min (90,84)}{\max (90,84)}$ & $\mathbf{0}$ \\
\hline
\end{tabular}

We have,

$T_{\left(d_{i}, d_{j}\right)}=\left(1-\frac{\min \left(d_{i}, d_{j}\right)}{\max \left(d_{i}, d_{j}\right)}\right)$ Where $\left\{\begin{array}{c}i \in[2, n] \text { Where } n \in \bullet \\ j \in[1, n-1] \text { Where } n \in \bullet\end{array}\right.$ 
So,

For $i=2$ we have:

For $j=1$ we have:

\begin{tabular}{|c|c|c|c|c|c|}
\hline \multicolumn{7}{|c|}{ Rainfall Matrix M(C) } \\
\hline & $\mathbf{8 0}$ & $\mathbf{8 2}$ & $\mathbf{8 1}$ & $\mathbf{8 4}$ & $\mathbf{9 0}$ \\
\hline $\mathbf{8 0}$ & $\mathbf{0}$ & $\#$ & $\#$ & $\#$ & $\#$ \\
\hline $\mathbf{8 2}$ & $\mathbf{0 . 0 2 4 4}$ & $\mathbf{0}$ & $\#$ & $\#$ & $\#$ \\
\hline $\mathbf{8 1}$ & $1-\frac{\min (81,80)}{\max (81,80)}$ & $1-\frac{\min (81,82)}{\max (81,82)}$ & $\mathbf{0}$ & $\#$ & $\#$ \\
\hline $\mathbf{8 4}$ & $1-\frac{\min (84,80)}{\max (84,80)}$ & $1-\frac{\min (84,82)}{\max (84,82)}$ & $1-\frac{\min (84,81)}{\max (84,81)}$ & $\mathbf{0}$ & $\#$ \\
\hline $\mathbf{9 0}$ & $1-\frac{\min (90,80)}{\max (90,80)}$ & $1-\frac{\min (90,82)}{\max (90,82)}$ & $1-\frac{\min (90,81)}{\max (90,81)}$ & $1-\frac{\min (90,84)}{\max (90,84)}$ & $\mathbf{0}$ \\
\hline
\end{tabular}

For $i=3$ we have:

For $j=1$ we have:

\begin{tabular}{|c|c|c|c|c|c|}
\hline \multicolumn{7}{|c|}{ Rainfall Matrix M(C) } \\
\hline $\mathbf{8 0}$ & $\mathbf{8 0}$ & $\mathbf{8 2}$ & $\mathbf{8 1}$ & $\mathbf{8 4}$ & $\mathbf{9 0}$ \\
\hline $\mathbf{8 2}$ & $\mathbf{0 . 0 2 4 4}$ & $\mathbf{0}$ & $\#$ & $\#$ & $\#$ \\
\hline $\mathbf{8 1}$ & $\mathbf{0 . 0 1 2 3}$ & $1-\frac{\min (81,82)}{\max (81,82)}$ & $\mathbf{0}$ & $\#$ & $\#$ \\
\hline $\mathbf{8 4}$ & $1-\frac{\min (84,80)}{\max (84,80)}$ & $1-\frac{\min (84,82)}{\max (84,82)}$ & $1-\frac{\min (84,81)}{\max (84,81)}$ & $\mathbf{0}$ & $\#$ \\
\hline $\mathbf{9 0}$ & $1-\frac{\min (90,80)}{\max (90,80)}$ & $1-\frac{\min (90,82)}{\max (90,82)}$ & $1-\frac{\min (90,81)}{\max (90,81)}$ & $1-\frac{\min (90,84)}{\max (90,84)}$ & $\mathbf{0}$ \\
\hline
\end{tabular}

For $i=3$ we have:

For $j=2$ we have:

\begin{tabular}{|c|c|c|c|c|c|}
\hline \multicolumn{7}{|c|}{ Rainfall Matrix M(C) } \\
\hline & $\mathbf{8 0}$ & $\mathbf{8 2}$ & $\mathbf{8 1}$ & $\mathbf{8 4}$ & $\mathbf{9 0}$ \\
\hline $\mathbf{8 0}$ & $\mathbf{0}$ & $\#$ & $\#$ & $\#$ & $\#$ \\
\hline $\mathbf{8 2}$ & $\mathbf{0 . 0 2 4 4}$ & $\mathbf{0}$ & $\#$ & $\#$ & $\#$ \\
\hline $\mathbf{8 1}$ & $\mathbf{0 . 0 1 2 3}$ & $\mathbf{0 . 0 1 2 2}$ & $\mathbf{0}$ & $\#$ & $\#$ \\
\hline $\mathbf{8 4}$ & $1-\frac{\min (84,80)}{\max (84,80)}$ & $1-\frac{\min (84,82)}{\max (84,82)}$ & $1-\frac{\min (84,81)}{\max (84,81)}$ & $\mathbf{0}$ & $\#$ \\
\hline $\mathbf{9 0}$ & $1-\frac{\min (90,80)}{\max (90,80)}$ & $1-\frac{\min (90,82)}{\max (90,82)}$ & $1-\frac{\min (90,81)}{\max (90,81)}$ & $1-\frac{\min (90,84)}{\max (90,84)}$ & $\mathbf{0}$ \\
\hline
\end{tabular}


For $i=4$ we have:

For $j=1$ we have:

\begin{tabular}{|c|c|c|c|c|c|}
\hline \multicolumn{7}{|c|}{ Rainfall Matrix M(C) } \\
\hline & $\mathbf{8 0}$ & $\mathbf{8 2}$ & $\mathbf{8 1}$ & $\mathbf{8 4}$ & $\mathbf{9 0}$ \\
\hline $\mathbf{8 0}$ & $\mathbf{0}$ & $\#$ & $\#$ & $\#$ & $\#$ \\
\hline $\mathbf{8 2}$ & $\mathbf{0 . 0 2 4 4}$ & $\mathbf{0}$ & $\#$ & $\#$ & $\#$ \\
\hline $\mathbf{8 1}$ & $\mathbf{0 . 0 1 2 3}$ & $\mathbf{0 . 0 1 2 2}$ & $\mathbf{0}$ & $\#$ & $\#$ \\
\hline $\mathbf{8 4}$ & $\mathbf{0 . 0 4 7 6}$ & $1-\frac{\min (84,82)}{\max (84,82)}$ & $1-\frac{\min (84,81)}{\max (84,81)}$ & $\mathbf{0}$ & $\#$ \\
\hline $\mathbf{9 0}$ & $1-\frac{\min (90,80)}{\max (90,80)}$ & $1-\frac{\min (90,82)}{\max (90,82)}$ & $1-\frac{\min (90,81)}{\max (90,81)}$ & $1-\frac{\min (90,84)}{\max (90,84)}$ & $\mathbf{0}$ \\
\hline
\end{tabular}

For $i=4$ we have:

For $j=2$ we have:

\begin{tabular}{|c|c|c|c|c|c|}
\hline \multicolumn{7}{|c|}{ Rainfall Matrix M(C) } \\
\hline & $\mathbf{8 0}$ & $\mathbf{8 2}$ & $\mathbf{8 1}$ & $\mathbf{8 4}$ & $\mathbf{9 0}$ \\
\hline $\mathbf{8 0}$ & $\mathbf{0}$ & $\#$ & $\#$ & $\#$ & $\#$ \\
\hline $\mathbf{8 2}$ & $\mathbf{0 . 0 2 4 4}$ & $\mathbf{0}$ & $\#$ & $\#$ & $\#$ \\
\hline $\mathbf{8 1}$ & $\mathbf{0 . 0 1 2 3}$ & $\mathbf{0 . 0 1 2 2}$ & $\mathbf{0}$ & $\#$ & $\#$ \\
\hline $\mathbf{8 4}$ & $\mathbf{0 . 0 4 7 6}$ & $\mathbf{0 . 0 2 3 8}$ & $1-\frac{\min (84,81)}{\max (84,81)}$ & $\mathbf{0}$ & $\#$ \\
\hline $\mathbf{9 0}$ & $1-\frac{\min (90,80)}{\max (90,80)}$ & $1-\frac{\min (90,82)}{\max (90,82)}$ & $1-\frac{\min (90,81)}{\max (90,81)}$ & $1-\frac{\min (90,84)}{\max (90,84)}$ & $\mathbf{0}$ \\
\hline
\end{tabular}

For $i=4$ we have:

For $j=3$ we have:

\begin{tabular}{|c|c|c|c|c|c|}
\hline \multicolumn{7}{|c|}{ Rainfall Matrix M(C) } \\
\hline & $\mathbf{8 0}$ & $\mathbf{8 2}$ & $\mathbf{8 1}$ & $\mathbf{8 4}$ & $\mathbf{9 0}$ \\
\hline $\mathbf{8 0}$ & $\mathbf{0}$ & $\#$ & $\#$ & $\#$ & $\#$ \\
\hline $\mathbf{8 2}$ & $\mathbf{0 . 0 2 4 4}$ & $\mathbf{0}$ & $\#$ & $\#$ & $\#$ \\
\hline $\mathbf{8 1}$ & $\mathbf{0 . 0 1 2 3}$ & $\mathbf{0 . 0 1 2 2}$ & $\mathbf{0}$ & $\#$ & $\#$ \\
\hline $\mathbf{8 4}$ & $\mathbf{0 . 0 4 7 6}$ & $\mathbf{0 . 0 2 3 8}$ & $\mathbf{0 . 0 3 5 7}$ & $\mathbf{0}$ & $\#$ \\
\hline $\mathbf{9 0}$ & $1-\frac{\min (90,80)}{\max (90,80)}$ & $1-\frac{\min (90,82)}{\max (90,82)}$ & $1-\frac{\min (90,81)}{\max (90,81)}$ & $1-\frac{\min (90,84)}{\max (90,84)}$ & $\mathbf{0}$ \\
\hline
\end{tabular}

For $i=5$ we have:

For $j=1$ we have:

\begin{tabular}{|c|c|c|c|c|c|}
\hline \multicolumn{7}{|c|}{ Rainfall Matrix M(C) } \\
\hline & $\mathbf{8 0}$ & $\mathbf{8 2}$ & $\mathbf{8 1}$ & $\mathbf{8 4}$ & $\mathbf{9 0}$ \\
\hline $\mathbf{8 0}$ & $\mathbf{0}$ & $\#$ & $\#$ & $\#$ & $\#$ \\
\hline $\mathbf{8 2}$ & $\mathbf{0 . 0 2 4 4}$ & $\mathbf{0}$ & $\#$ & $\#$ & $\#$ \\
\hline $\mathbf{8 1}$ & $\mathbf{0 . 0 1 2 3}$ & $\mathbf{0 . 0 1 2 2}$ & $\mathbf{0}$ & $\#$ & $\#$ \\
\hline $\mathbf{8 4}$ & $\mathbf{0 . 0 4 7 6}$ & $\mathbf{0 . 0 2 3 8}$ & $\mathbf{0 . 0 3 5 7}$ & $\mathbf{0}$ & $\#$ \\
\hline $\mathbf{9 0}$ & $\mathbf{0 . 1 1 1 1}$ & $1-\frac{\min (90,82)}{\max (90,82)}$ & $1-\frac{\min (90,81)}{\max (90,81)}$ & $1-\frac{\min (90,84)}{\max (90,84)}$ & $\mathbf{0}$ \\
\hline
\end{tabular}


For $i=5$ we have:

For $j=2$ we have:

\begin{tabular}{|c|c|c|c|c|c|}
\hline \multicolumn{7}{|c|}{ Rainfall Matrix M(C) } \\
\hline & $\mathbf{8 0}$ & $\mathbf{8 2}$ & $\mathbf{8 1}$ & $\mathbf{8 4}$ & $\mathbf{9 0}$ \\
\hline $\mathbf{8 0}$ & $\mathbf{0}$ & $\#$ & $\#$ & $\#$ & $\#$ \\
\hline $\mathbf{8 2}$ & $\mathbf{0 . 0 2 4 4}$ & $\mathbf{0}$ & $\#$ & $\#$ & $\#$ \\
\hline $\mathbf{8 1}$ & $\mathbf{0 . 0 1 2 3}$ & $\mathbf{0 . 0 1 2 2}$ & $\mathbf{0}$ & $\#$ & $\#$ \\
\hline $\mathbf{8 4}$ & $\mathbf{0 . 0 4 7 6}$ & $\mathbf{0 . 0 2 3 8}$ & $\mathbf{0 . 0 3 5 7}$ & $\mathbf{0}$ & $\#$ \\
\hline $\mathbf{9 0}$ & $\mathbf{0 . 1 1 1 1}$ & $\mathbf{0 . 0 8 8 8}$ & $1-\frac{\min (90,81)}{\max (90,81)}$ & $1-\frac{\min (90,84)}{\max (90,84)}$ & $\mathbf{0}$ \\
\hline
\end{tabular}

For $i=5$ we have:

For $j=3$ we have:

\begin{tabular}{|c|c|c|c|c|c|}
\hline \multicolumn{7}{|c|}{ Rainfall Matrix M(C) } \\
\hline & $\mathbf{8 0}$ & $\mathbf{8 2}$ & $\mathbf{8 1}$ & $\mathbf{8 4}$ & $\mathbf{9 0}$ \\
\hline $\mathbf{8 0}$ & $\mathbf{0}$ & $\#$ & $\#$ & $\#$ & $\#$ \\
\hline $\mathbf{8 2}$ & $\mathbf{0 . 0 2 4 4}$ & $\mathbf{0}$ & $\#$ & $\#$ & $\#$ \\
\hline $\mathbf{8 1}$ & $\mathbf{0 . 0 1 2 3}$ & $\mathbf{0 . 0 1 2 2}$ & $\mathbf{0}$ & $\#$ & $\#$ \\
\hline $\mathbf{8 4}$ & $\mathbf{0 . 0 4 7 6}$ & $\mathbf{0 . 0 2 3 8}$ & $\mathbf{0 . 0 3 5 7}$ & $\mathbf{0}$ & $\#$ \\
\hline $\mathbf{9 0}$ & $\mathbf{0 . 1 1 1 1}$ & $\mathbf{0 . 0 8 8 8}$ & $\mathbf{0 . 1}$ & $1-\frac{\min (90,84)}{\max (90,84)}$ & $\mathbf{0}$ \\
\hline
\end{tabular}

Here is the final resulting matrix.

\begin{tabular}{|l|c|c|c|c|c|}
\hline \multicolumn{7}{|c|}{ Rainfall Matrix M(C) } \\
\hline & $\mathbf{8 0}$ & $\mathbf{8 2}$ & $\mathbf{8 1}$ & $\mathbf{8 4}$ & $\mathbf{9 0}$ \\
\hline $\mathbf{8 0}$ & $\mathbf{0}$ & $\#$ & $\#$ & $\#$ & $\#$ \\
\hline $\mathbf{8 2}$ & $\mathbf{0 . 0 2 4 4}$ & $\mathbf{0}$ & $\#$ & $\#$ & $\#$ \\
\hline $\mathbf{8 1}$ & $\mathbf{0 . 0 1 2 3}$ & $\mathbf{0 . 0 1 2 2}$ & $\mathbf{0}$ & $\#$ & $\#$ \\
\hline $\mathbf{8 4}$ & $\mathbf{0 . 0 4 7 6}$ & $\mathbf{0 . 0 2 3 8}$ & $\mathbf{0 . 0 3 5 7}$ & $\mathbf{0}$ & $\#$ \\
\hline $\mathbf{9 0}$ & $\mathbf{0 . 1 1 1 1}$ & $\mathbf{0 . 0 8 8 8}$ & $\mathbf{0 . 1}$ & $\mathbf{0 . 0 6 6 7}$ & $\mathbf{0}$ \\
\hline
\end{tabular}

$\Delta_{C}=1.2$ for the sensor used in forecasting that is installed in the area under observation, and $T_{F}=5$ according to a study carried out in several areas in Morocco for the rainfall factor, so $P_{T C}=0.074$. Thus, after completing several experiments, we found that when we do a comparison between the tolerance percentage and the matrix elements already calculated inside a loop statement for all the sensors data, we obtain the data that are valid and the data that are invalid in order to be stored in the database.

If $P_{T C}-T\left(d_{i}, d_{j}\right) \geq 0$, then the data sent by this sensor is valid else, it is invalid data. Therefore, the valid data for the Rainfall Matrix $\mathrm{M}(\mathrm{C})$ above are 
ValidData $_{\text {Rainfall }}=\{80,82,81,84\}$ and the invalid data for the Rainfall Matrix $\mathrm{M}(\mathrm{C})$ above are: InvalidData $a_{\text {Rainfall }}=\{90\}$

Additionally, the proposed model performs the same process for the other vectors (Runoff and Water Level).

When all of the agents have completed their processing and the data classification and aggregation steps have been completed successfully, each agent sends ACL messages to the database interaction agent to trigger the database interaction stage.

\subsection{Database Interaction Stage}

Before saving data in the database, the agent performs additional processing. First, for the valid data, it calculates the average of the data, then it calculates the distance between the average and the data, and, finally, the agent selects the data that has the nearest distance to the average and saves it as the most relevant data.

Second, the invalid data are saved in the database, and the agent calculates the number of mistakes made by the sensors that sent invalid data. When a sensor exceeds five errors, it turns off immediately because it requires maintenance or replacement. This number of errors parameter is configured from the platform for indicating it as needed.

\subsection{Algorithm of the proposed model}

In this section, we will present the algorithm of our proposed model. The functions VerificationFunctionalSensors(), VerificationNonFunctionalSensors) are used to search for the sensors that are functional and those that are not and to arrange them in lists to be used by the main algorithm, respectively. The SensorVerificationAgent() function is used to display the list of functional and non-functional sensors and to send warning messages, text messages and emails to the managers to repair or replace the sensors that are non-functional..

The SavingFinalValidData() procedure is used to calculate the average of all of the valid data, to compare these data with the average using comparisons of the distances between the data and the average, then to take the nearest data from average and, finally, to save the most appropriate data in the database. The procedures SavingNonValidData(), ErroneousNumberTime() and ChangeFlag() are used to save invalid data in the database, to increment the number of errors given by the sensor and to change the statuses of sensors from active to inactive if they exceed the number of errors that we set, which is five by default, respectively. The presented algorithm is responsible for performing the classification and aggregation of the data received from the wireless sensors using the proposed model, including the functions and procedures presented above. The algorithm is presented below. 
Function VerificationFunctionalSensors(Flag)

\section{Data:}

List SensorsFunctionalList;

\section{Begin}

SensorsFunctionalList $=$ ResearchFunctionalSensorsDB(Flag); return SensorsFunctionalList;

\section{End}

Function VerificationNonFunctionalSensors(Flag)

\section{Data:}

List SensorsNFunctionalList;

\section{Begin}

SensorsNFunctionalList $=$ ResearchNonFunctionalSensorsDB(Flag); return SensorsNFunctionalList;

\section{End}

Procedure SavingFinalValidData(ValidDataList)

Data:

Double Average;

Double FinalValidData;

\section{Begin}

Average $=$ CalculateAverageDataValidList $($ ValidDataList $)$;

FinalValidData $\quad=\quad$ CalculateSmallestDistanceAverageData(ValidDataList,Average);

SavingDB(SensorID, FinalValidData);

End 
Procedure SavingNonValidData(NonValidDataList)

\section{Begin}

SavingDB(SensorIDList, NonValidDataList);

ErroneousNumberTime(SensorIDList);

End

Procedure ErroneousNumberTime(SensorIDList)

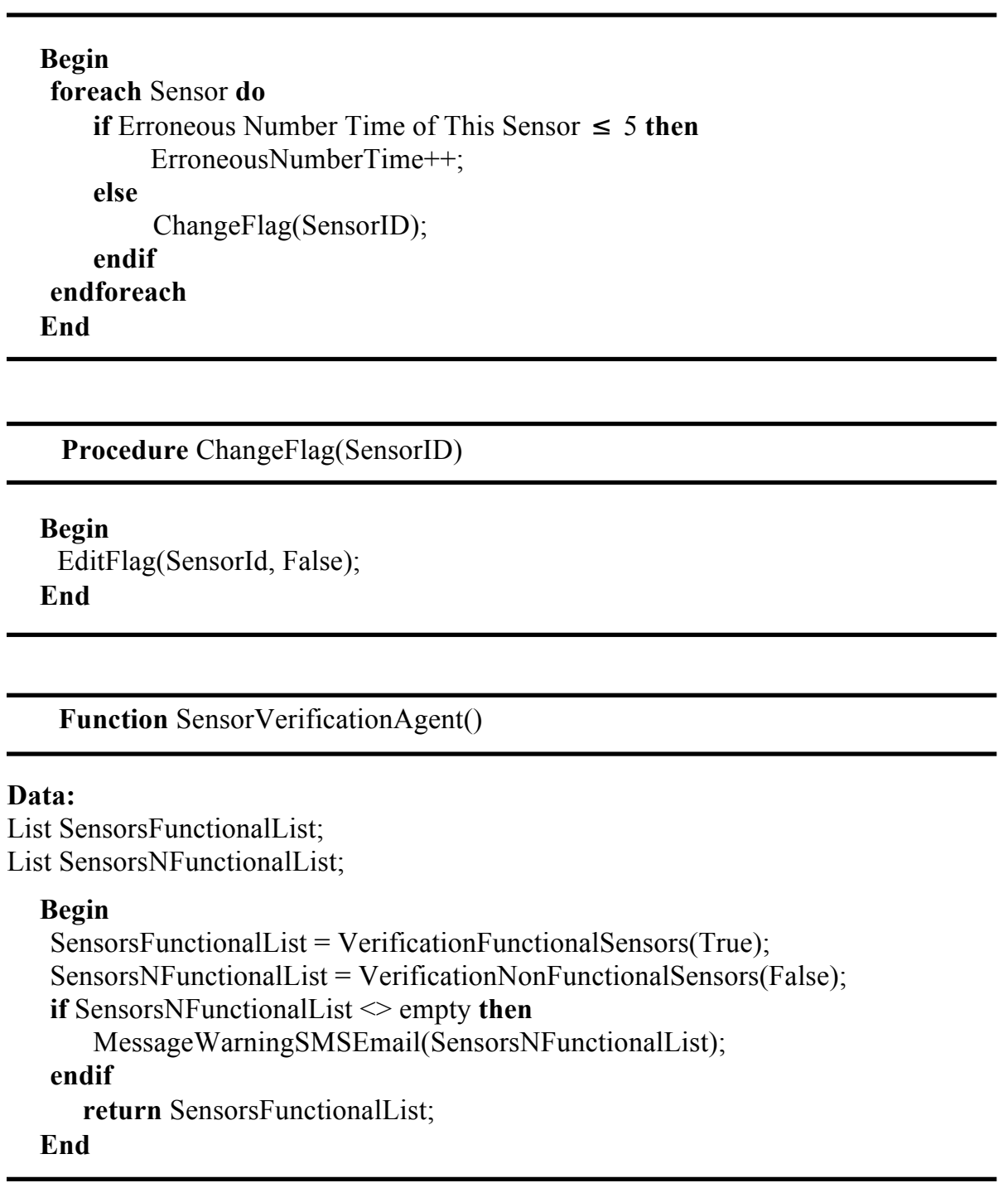


Procedure DatabaseInteractionAgent(ValidDataList, NonValidDataList)

\section{Begin}

SavingFinalValidData(ValidDataList);

SavingNonValidData(NonValidDataList);

End

Algorithm DataClassificationAggregationAgentSensors

\section{Data:}

List FunctionalDataList;

List ValidDataList;

List NonValidDataList;

List DataSensorFileList;

Matrix M;

Double $P_{T C}$;

Double $\Delta_{C}, T_{F}$;

\section{Begin}

The verification agent function Triggers the process of the classification using ACL Message and send the SensorsFunctionalList;

if SensorsFunctionalList $<>$ empty then

DataSensorFileList $=$ ReadFile(File);

foreach Functional Sensor from DataSensorFileList do

FunctionalDataList $=$ TempList[DataSensorFunctional];

endforeach

for $i=2$ To FunctionalDataList.size ()$-1$ do

for $j=1$ To $i$ do

$$
M[i, j]=1-\frac{\operatorname{Min}(\text { ValidDataList }[i], \text { ValidDataList }[j])}{\operatorname{Max}(\text { ValidDataList }[i], \text { ValidDataList }[j])} ;
$$

endfor

endfor

$P_{T C}=\frac{\left(2 \times \Delta_{C}\right)+T_{F}}{100} ;$

for $i=2$ To FunctionalDataList.size ()$-1$ do

for $j=1$ To $i$ do

if $P_{T C}-M[i, j] \geq 0$ then

if This sensor does't exist in the list then

ValidDataList[i] = FunctionalDataList[i]; 


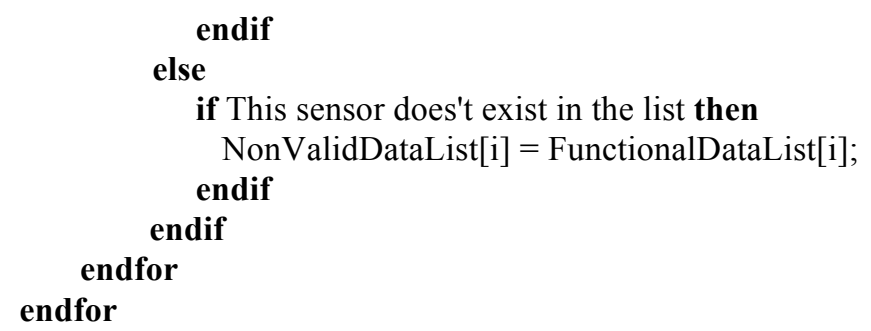

The three agents trigger the database interaction stage by sending ACL message and the valid and erroneous list to the database interaction agent; endif

End

\subsection{Pre-Processing Mode Architecture}

The figure 1 below presents the architecture of the pre-processing mode.

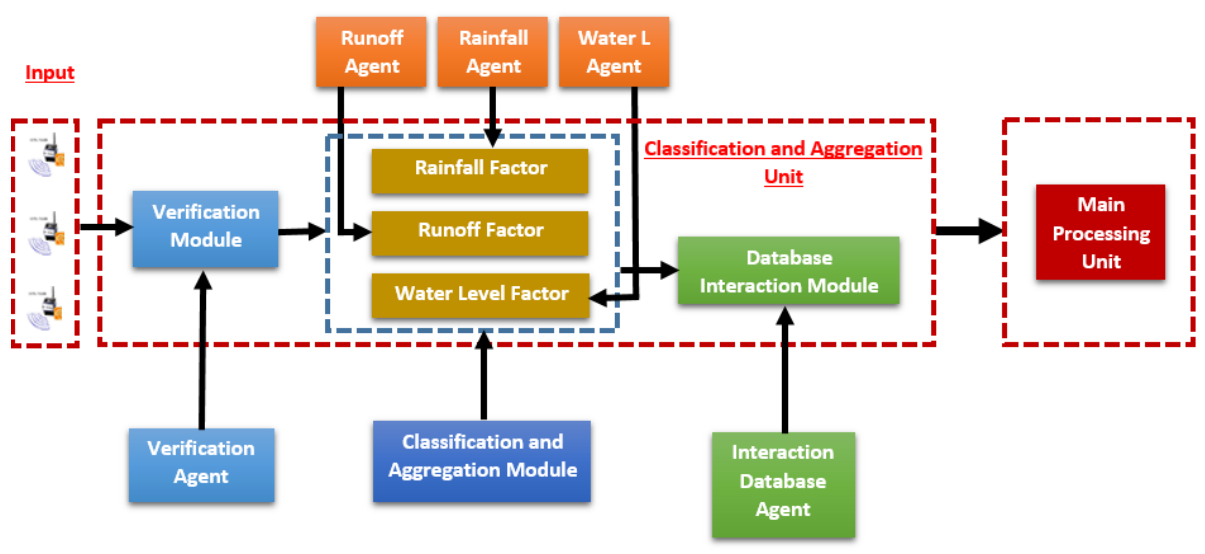

Fig. 1. Pre-Processing Mode Architecture

\section{Design \& Implementation}

For system design, we chose visual modeling as a design model for this system because of its many benefits regarding simplicity, universality, conciseness and expressiveness. The language used is the UML, which is a graphical language for modeling data and processing. The modeling was performed on three levels, structural modeling, behavioral modeling and interaction between objects [18]. 


\subsection{Behavioral modeling}

Behavioral diagrams focus on the dynamic behavior of the system, the behavioral diagrams that we used are the use case diagram and sequence diagram. The main actors in our system are the decision makers who watch over the control and management of floods, responsibles and administrators. The use case diagrams are shown in figure 2.

The sequence diagrams are shown in the figures 3 .

\subsection{Interaction Modeling}

The diagrams of interaction between objects modeling used to model the dynamic behavior of the system, and to indicate how the objects interact at run time. The most interesting diagram of this kind of modeling is the communication diagram. The communication diagrams are shown in the figure 4.

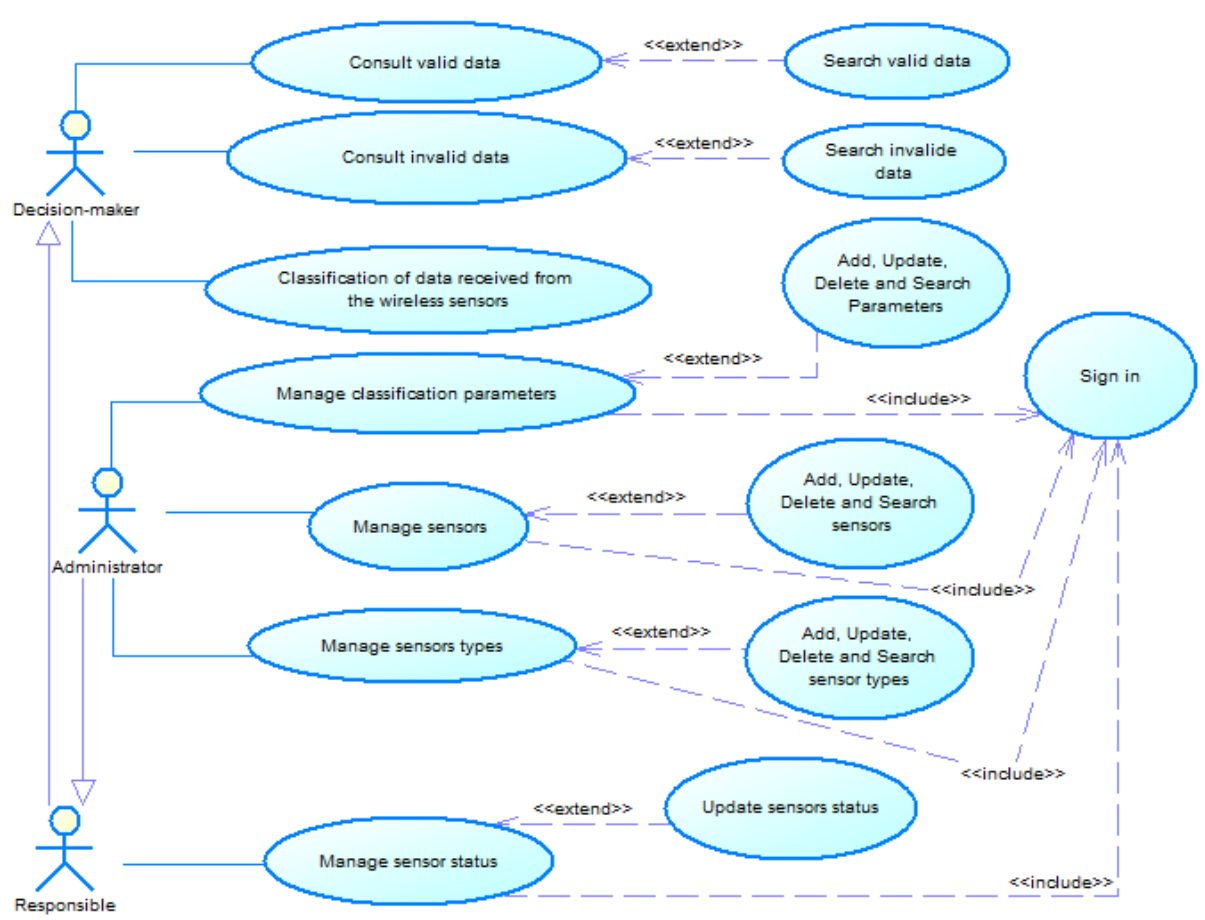

Fig. 2. Use cas diagram for Pre-Processing phase 
Paper-Proposed Intelligent Pre-Processing Model of Real-Time Flood Forecasting and Warning for Dat..

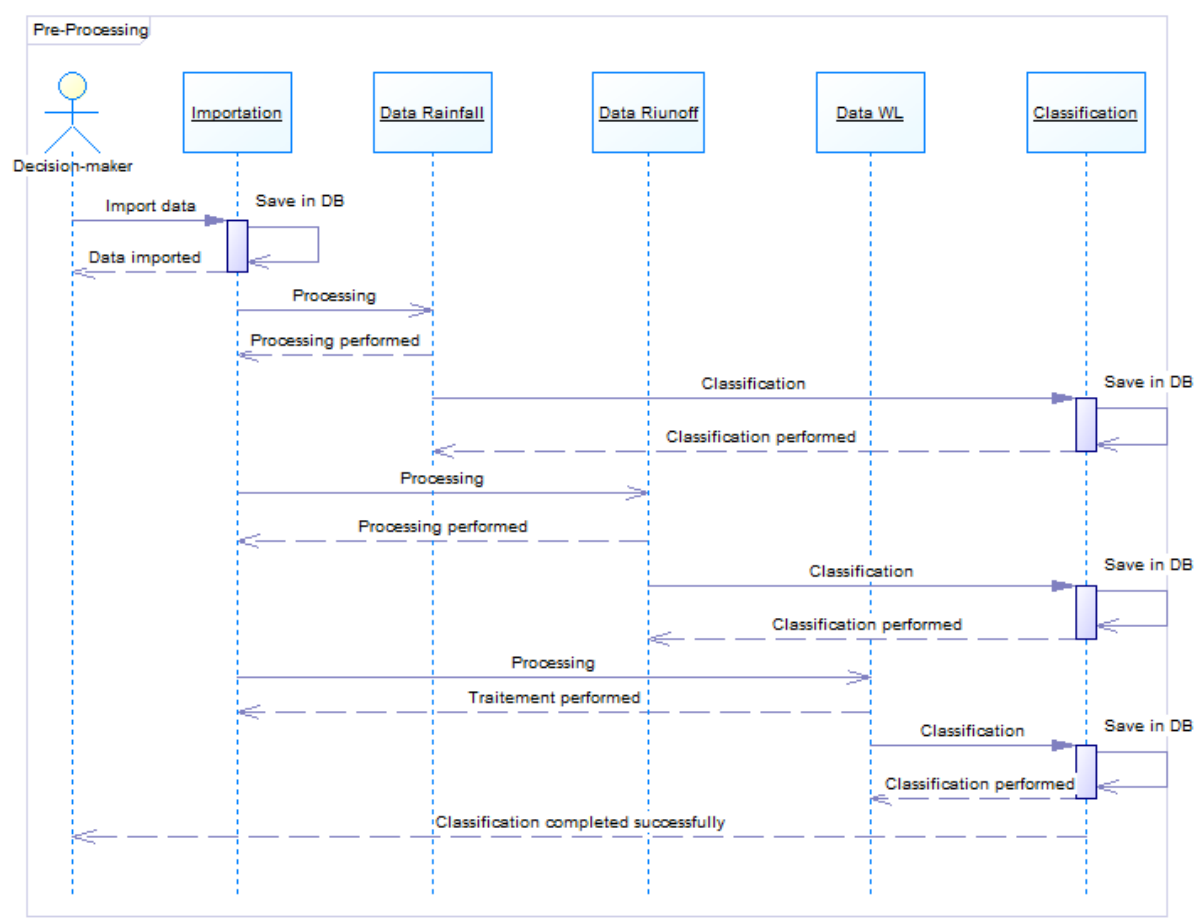

Fig. 3. Sequence diagram for Main Processing phase

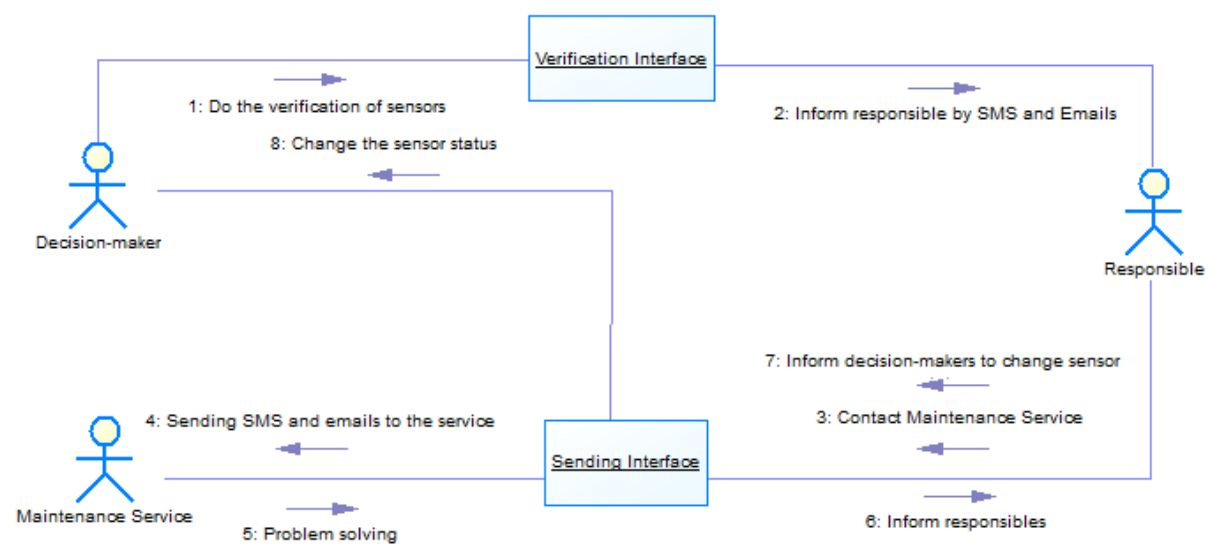

Fig. 4. Communication diagram for Pre-Processing phase 


\subsection{Implementation}

In this section, we present our Intelligent Pre-Processing Model of Real-Time Flood Forecasting and Warning for Data Classification and Aggregation.

In this stage, as already described, the system first verifies the functioning of the wireless sensors installed in the surveillance area to identify the functioning and nonfunctioning sensors. The system disables the wireless sensors that are broken to remove them from the forecasting process and to have them repaired later. This is the warning message from the system show in the figure 5:

The Sensors entitled ( Sensor_Rainfall_5 Sensor_Flow_Velocity_1 Sensor_Water_Level_3 ) are broken down, it must be repaired ! Call the maintenance service immediately

Fig. 5. Sensors verification phase

Next Figures 6, 7 and 8 presents the classification interfaces of the proposed model for classification and aggregation, where the most appropriate value to be stored in the database is obtained from all of the valid data. In this stage, we also obtain the invalid values and the sensors that sent the erroneous values.

The figures $9,10,11$ and 12 present the agents that perform the pre-processing in the JADE platform.

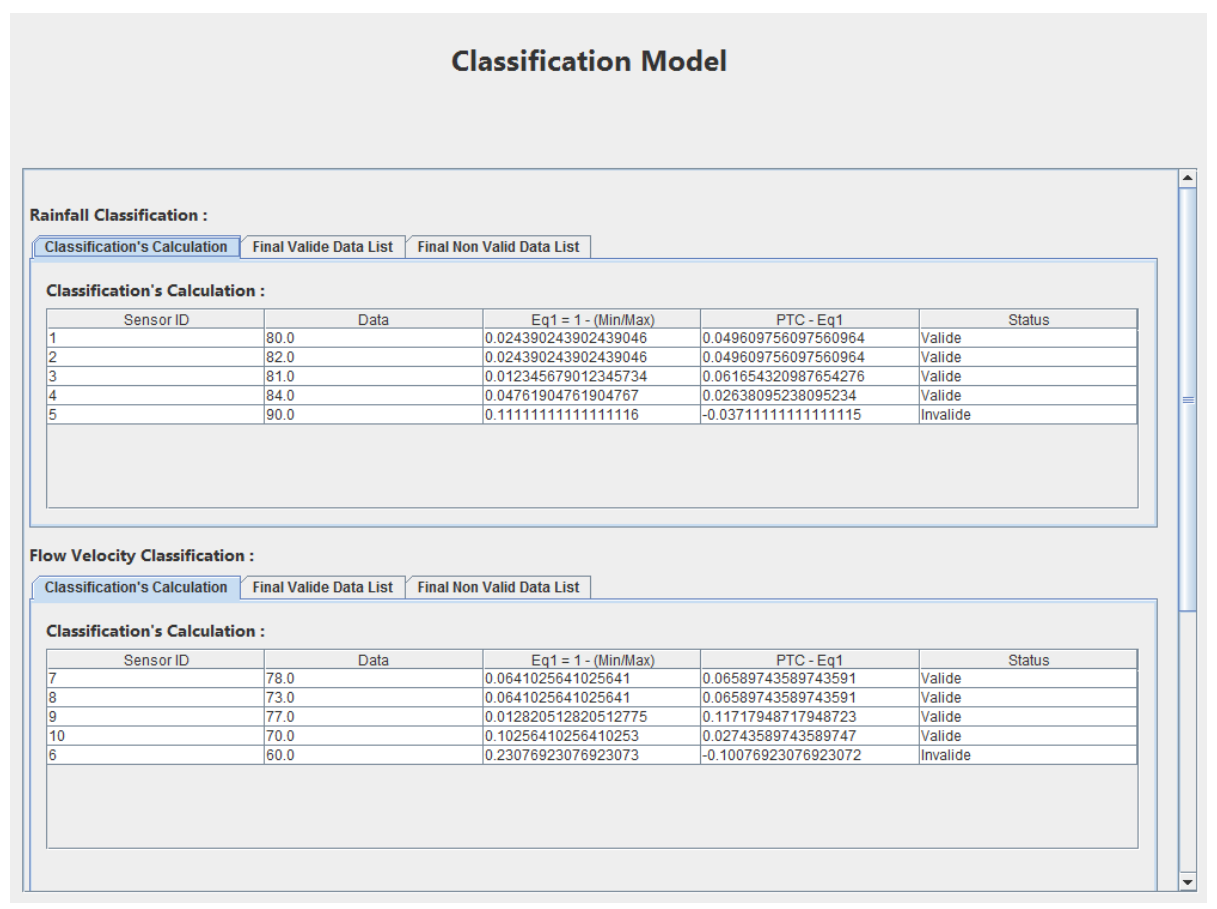

Fig. 6. Classification and aggregation process by the proposed model 


\section{Classification Model}

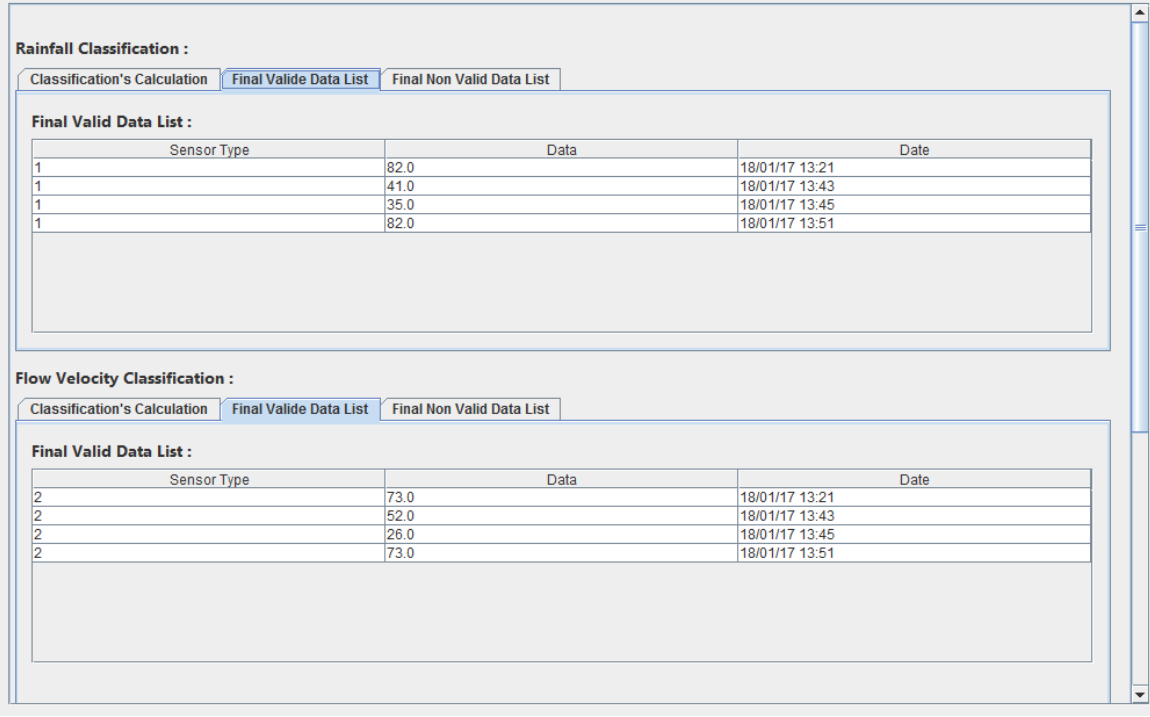

Fig. 7. List of final valid data

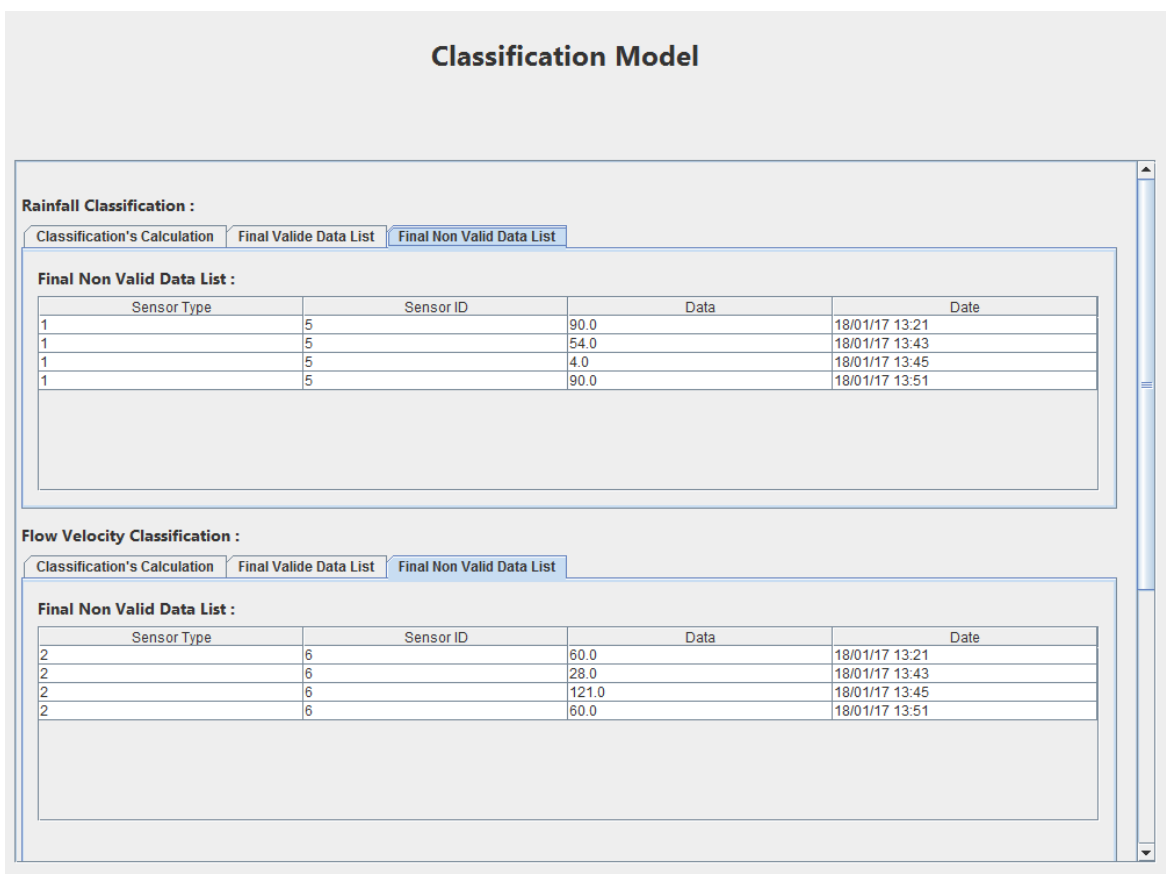

Fig. 8. List of invalid data 


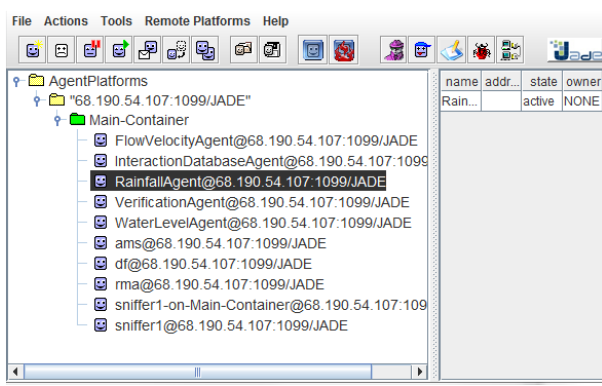

Fig. 9. Agents in JADE

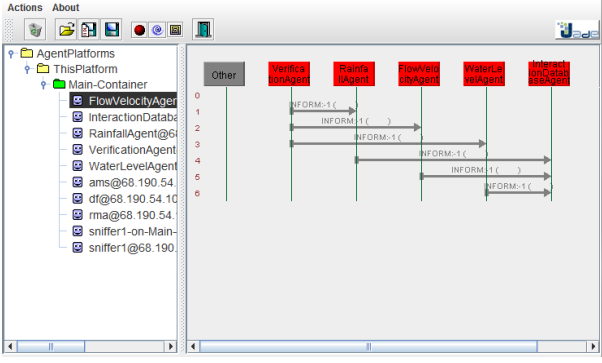

Fig. 11. Classification Phase

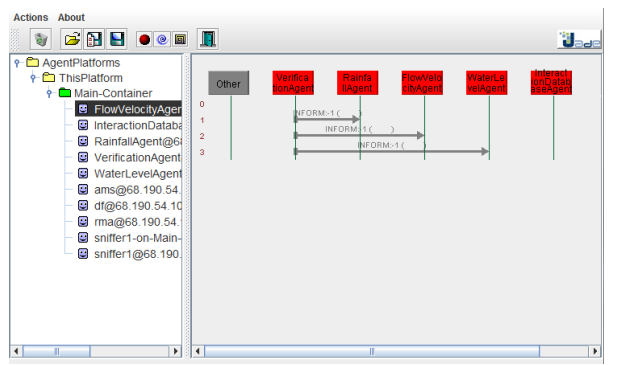

Fig. 10. Verification Phase

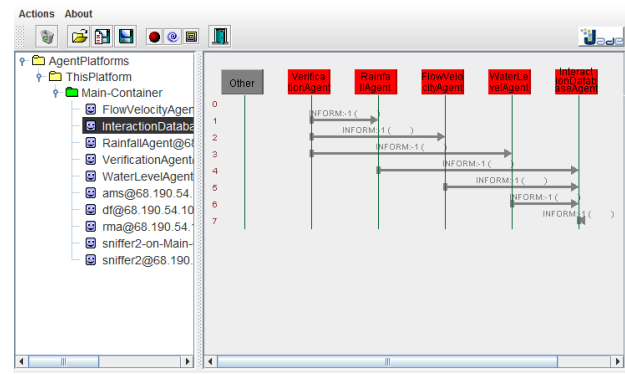

Fig. 12. Interaction with database phase

\section{$5 \quad$ Results \& Discussion}

To evaluate the performance of our system and our different proposed models, we made several experiments. For the Pre-Processing mode, we are interested to the accuracy criterion of its performance and its ability to classify data. To evaluate the performance and the accuracy, we have done forty-five experiments; in each experiment, we increased the number of data to classify in order to test the performance of our systems, we used the following formula:

$$
\text { Accuracy }=\frac{F V D}{F V D+F I V D}
$$

Where:

FVD: is the Final Valid Data filtered after the classification process by our proposed model for classification and aggregation.

FIVD: is the Final InValid Data filtered after the classification process by our proposed model for classification and aggregation.

During experiments, we noticed that the classification performance of our proposed model decreases when we increase the number of data. In the last experiment, we gave the system to classify 6126 data but it has classified only $2.5 \%$ of data. This disadvantage can never influence the performance of our distributed decision support system for real-time flood forecasting, because we have designed our system to take into account three parameters to make the forecasting and the warning. 
Three types of sensors installed in the at-risk areas are responsible for sending data. Each sensor has five copies, so the maximum number of data sent in real time to be classified is fifteen, so the results achieved already indicate that our system can classify $100 \%$ of the data until three hundred data are reached. Thus, for our system, the performance of the classifications for the Pre-Processing mode do not and will never decrease and the decision accuracy will always be excellent because we will never reach three hundred data sent in real time. Figure 13 summarizes what we have just explained.

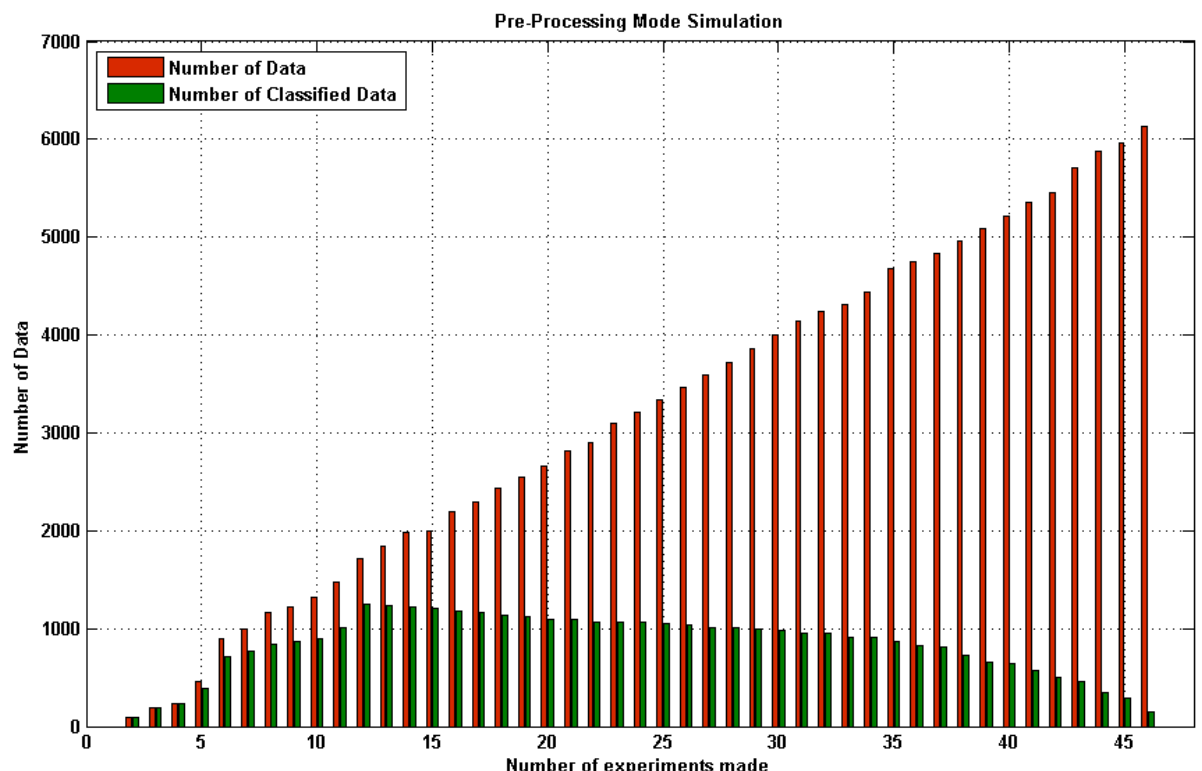

Fig. 13. Pre-Processing Mode Simulation

\section{Conclusions}

In this paper, we presented a new proposed intelligent pre-processing model of real-time flood forecasting and warning data received from the wireless sensors in order to do the classification and the aggregation of data using multi-agents system. Our proposed model is divided into three stages:

- Sensor Verification Stage for monitoring the proper functioning of the wireless sensors

- Data Aggregation and Classification Stage for the classification and aggregation to get the most appropriate data from the wireless sensors in order to guarantee the accuracy of our data to have to best accurate decision from our decision support system for real-time flood forecasting and warning 
- Finally the Database Interaction Stage for saving the valid and the invalid data and to trigger the offline mode of our decision support system

Our future work will focus on the reducing energy consumption and extending the lifetime of the sensors by using the clustering approach to regroup sensors into clusters and selecting a cluster head, whom will communication with the base station. We will use also the multi-agent systems to speed up this process and to transform the wireless into a collaborative, cooperative and distributed physical wireless sensor network.

\section{$7 \quad$ References}

[1] G. Furquim et al., "Combining Wireless Sensor Networks and Machine Learning for Flash Flood Nowcasting," 2014 28th International Conference on Advanced Information Networking and Applications Workshops, Victoria, BC, 2014, pp. 67-72. https://doi.org/10.1109/WAINA.2014.21

[2] Sagar Sudhakar Mane and M. K. Mokashi, "Real-Time Flash-Flood Monitoring, Alerting and Forecasting System using Data Mining and wireless sensor Network," 2015 International Conference on Communications and Signal Processing (ICCSP), Melmaruvathur, 2015, pp. 1881-1886. https://doi.org/10.1109/ICCSP.2015.7322851

[3] T. Hashimoto, R. Ito, T. Ohta and Y. Kakuda, "Information Dissemination Scheme Using Mobile Agents in Autonomous Clustering-Based Heterogeneous Wireless Networks," 2016 Fourth International Symposium on Computing and Networking (CANDAR), Hiroshima, Japan, 2016, pp. 279-285. https://doi.org/10.1109/CANDAR.2016.0057

[4] S. V. Halde and S. T. Khot, "Efficient collection of big data in WSN," 2016 International Conference on Inventive Computation Technologies (ICICT), Coimbatore, India, 2016, pp. 1-5. https://doi.org/10.1109/INVENTIVE.2016.7823196

[5] F. Del Ama Gonzalo, J. A. Hernandez and B. Moreno, "Wireless low cost temperature and humidity sensors with PV microcells. Determination of design parameters by means of experimental measurements," 2016 5th International Conference on Electronic Devices, Systems and Applications (ICEDSA), Ras Al Khaimah, United Arab Emirates, 2016, pp. 1-4.

[6] Yenke Blaise Omer, Sambo Damien Wohwe, Ari Abo Adamou Abba and Gueroui Abdelhak " MMEDD: Multithreading Model for an Efficient Data Delivery in Wireless Sensor Networks," International Journal of Communication Networks and Information Security (IJCNIS), Vol 8, Issue 3, December 2016, pp. 179-186.

[7] R. I. Batygin and O. K. Alsova, "Software system for different types of data classification based on the ensemble algorithms," 2016 13th International Scientific-Technical Conference on Actual Problems of Electronics Instrument Engineering (APEIE), Novosibirsk, 2016, pp. 506-509.

[8] Y. Yan, Q. Zhu, M. L. Shyu and S. C. Chen, "A Classifier Ensemble Framework for Multimedia Big Data Classification," 2016 IEEE 17th International Conference on Information Reuse and Integration (IRI), Pittsburgh, PA, 2016, pp. 615-622.

[9] A. Hamzi, M. Koudil, J. Jamont and M. Occello, "Multi-Agent Architecture for the Design of WSN Applications," Wireless Sensor Network, Vol. 5 No. 2, 2013, pp. 14-25. https://doi.org/10.4236/wsn.2013.52003

[10] E. Belghache, J. P. Georgé and M. P. Gleizes, "Towards an Adaptive Multi-agent System for Dynamic Big Data Analytics," 2016 Intl IEEE Conferences on Ubiquitous Intelligence \& Computing, Advanced and Trusted Computing, Scalable Computing and Communica- 
tions, Cloud and Big Data Computing, Internet of People, and Smart World Congress (UIC/ATC/ScalCom/CBDCom/IoP/SmartWorld), Toulouse, France, 2016, pp. 753-758. https://doi.org/10.1109/UIC-ATC-ScalCom-CBDCom-IoP-SmartWorld.2016.0121

[11] C. Bennila Thangammal, P. Rangarajan and J. Raja Paul Perinban, "Low power wireless sensor networks for real-time data acquisition system using Bloom filter," 2011 International Conference on Emerging Trends in Electrical and Computer Technology, Tamil Nadu, 2011, pp. 88-91. https://doi.org/10.1109/ICETECT.2011.5760097

[12] A. Jorio, S. El Fkihi, B. Elbhiri and D. Aboutajdine, "A hierarchical clustering algorithm based on spectral classification for Wireless Sensor Networks," 2014 International Conference on Multimedia Computing and Systems (ICMCS), Marrakech, 2014, pp. 861-866.

[13] V. M. Kuthadi, R. Selvaraj and T. Marwala, "An efficient adaptive preprocessing mechanism for streaming sensor data," 2015 IEEE 9th International Conference on Intelligent Systems and Control (ISCO), Coimbatore, 2015, pp. 1-6.

[14] J. P. Yoon and J. Ortiz, "Data mining approach to situation-aware sensor actuation in wireless sensor networks," 2015 Fourth International Conference on Future Generation Communication Technology (FGCT), Luton, 2015, pp. 1-6. https://doi.org/10.1109/FGCT. $\underline{2015.7300250}$

[15] H. Ayadi, A. Zouinkhi, B. Boussaid and M. N. Abdelkrim, "A machine learning methods: Outlier detection in WSN," 2015 16th International Conference on Sciences and Techniques of Automatic Control and Computer Engineering (STA), Monastir, 2015, pp. 722727. https://doi.org/10.1109/STA.2015.7505190

[16] Amir Mehmood, Akhter Raza; Adnan Nadeem and Umair Saeed " Study of MultiClassification of Advanced Daily Life Activities on SHIMMER Sensor Dataset," International Journal of Communication Networks and Information Security (IJCNIS), Vol 8, Issue 2, August 2016, pp. 86-92.

[17] E. M. Marouane, E. Mostafa and E. Mohamed, "Intelligent data classification and aggregation in wireless sensors for flood forecasting system," Proceedings of 2014 Mediterranean Microwave Symposium (MMS2014), Marrakech, 2014, pp. 1-8. https://doi.org/10.1109/ MMS.2014.7088991

[18] El Mabrouk Marouane, Ezziyyani Mostafa, SADOUQ A. Zouahir and Essaaidi Mohammad " New Expert System for Short, Medium and Long term Flood Forecasting and Warning, " Journal of Theoretical and Applied Information Technology (JATIT), Vol 78, Issue 2, August 2015, pp. 286-302.

\section{Authors}

Marouane EL MABROUK is a doctor and researcher in the Mathematics and Application Laboratory, reviewer and committee member in several journals and conferences, IEEE Member and holder of $\mathrm{PhD}$ from the Faculty of Sciences and Technologies, Abdelmalek Essaadi University, Tangier, Morocco.

Salma GAOU is a doctor and researcher in LIROSA Laboratory, IEEE Member and holder of $\mathrm{PhD}$ from the Faculty of sciences, Abdelmalek Esaâdi University, Tetuan, Morocco.

Article submitted 04 July 2017. Published as resubmitted by the authors 15 September 2017. 